\title{
Voltage distribution of a wind turbine transformer under direct lightning strike: effects of the grounding of cable shield
}

\begin{abstract}
In this paper we consider wind energy generators as tall structures, thus the reflections of the current of a direct lightning strike, at various interfaces, have been included in the computational model. A significant effect of such reflections on the voltage distribution has been observed thus, inclusion of the reflections in the computation model has been justified. The study focuses on over voltage at critical locations that have been reported in the literature as having high probability of lightning damage. All parts related to the power generation in a wind turbine, including the step up transformer are modeled by lumped parameters using the Matlab/Simulink software. The transient response is obtained by applying the lightning impulse current to the equivalent circuit under several values of grounding resistance and several cable sheath grounding methods with different lightning strike waveforms. The results show that the location of the cable shield grounding and the grounding resistance values have significant influence on the produced over voltage waveforms at the input and output of transformer terminals. Appropriate surge protection devices for avoiding dangerous over voltage are proposed to reduce harmful effects.
\end{abstract}

Keyword: Lightning; Over voltage protection; Transformer; Transients; Wind turbine 